\title{
Optimal UAV Coordination for Target Tracking using Dynamic Programming
}

\author{
Steven A. P. Quintero, Francesco Papi, Daniel J. Klein, Luigi Chisci, and João P. Hespanha
}

\begin{abstract}
This work focuses on optimal routing for two camera-equipped UAVs cooperatively tracking a single target moving on the ground. The UAVs are small fixed-wing aircraft cruising at a constant speed and fixed altitude; consequently, the vehicles are modeled as planar Dubins vehicles. A perspective transformation, relating the image-plane measurements to the ground, allows derivation of the geolocation (target localization) error covariance. Using dynamic programming, we compute optimal coordinated control policies which minimize the fused geolocation error covariance. A surprising result, and the main contribution of this work, is that the dominant factor governing the optimal UAV routes is coordination of the distances to the target, not of the viewing directions as is traditionally assumed.
\end{abstract}

\section{INTRODUCTION}

In recent years, small unmanned aerial vehicles (UAVs) have found application in tasks such as surveillance, search and rescue, mapping, and real-time monitoring. We focus on the problem of optimally coordinating the motions of two UAVs for the purpose of tracking a target vehicle moving on the ground. Each UAV flies at a fixed altitude, and is equipped with a global positioning system (GPS), an inertial navigation system (INS), and a gimbaled video camera.

To track objects on the ground, video processing algorithms are used to determine the centroid pixel coordinates of targets that are moving in the image frame [1]. Given these pixel coordinates, the intrinsic and extrinsic camera parameters, and the terrain data, one can estimate the threedimensional location of the target in inertial coordinates and compute the associated error covariance. This is the process of geolocation for video cameras.

The geolocation error covariance is highly sensitive to the relative position of the UAVs with respect to the target. When a UAV is far from the target, relative to its height above the target, the resulting error covariance is significantly elongated in the viewing direction. The smallest geolocation error covariance comes when the UAV is directly above the target, in which case the error covariance is circular. Thus, the UAVs would ideally hover directly above the target, but the UAV dynamics preclude this viewing position from being maintained over a period of time.

Much research has proposed coordinated target tracking controllers without explicitly considering estimation of the target. For two UAVs, a good practice is to have the UAVs

Steven A. P. Quintero, Daniel J. Klein, and João P. Hespanha, Department of Electrical and Computer Engineering, University of California, Santa Barbara, CA 93106-9560, USA, squintero@umail.ucsb.edu, \{djklein, hespanha\}@ece.ucsb.edu.

Francesco Papi and Luigi Chisci, Dip. Sistemi e Informatica, Università degli Studi di Firenze, Florence, Italy, fpapi.unifi@gmail.com, chisciedsi.unifi.it. orbit the target at a nominal standoff distance and maintain an angular separation of $90^{\circ}$. This $90^{\circ}$ angle separation minimizes the instantaneous geolocation error covariance as the error ellipses are orthogonal [11]. While it is not possible to continually maintain a $90^{\circ}$ angle separation with fixedspeed aircraft, much work has been dedicated to designing controllers that achieve this goal approximately, see [5] and [8] for example. Others have developed methods to achieve and maintain diverse viewing angles, [4], [7]. In [6], Kingston develop a coordinated controller in which the UAVs orbit the target periodically, with phases spread uniformly (splay) in time, while maintaining a fixed standoff distance.

In each of the studies discussed above, the control laws are designed to produce a coordinated behavior that should result in improved geolocation estimates as compared to uncoordinated behaviors (without explicitly considering geolocation). Recent work by Stachura et al. [10] has optimized a simplified form of the geolocation error covariance for two UAVs using bearing-only sensors in the presence of communication packet losses. The controller is of the online receding horizon type. In this work, the UAVs are restricted to orbit the target at a nominal standoff distance, in which case maintaining the $90^{\circ}$ separation angle is an important factor.

Our work is optimization-based, like the work mentioned above, but differs significantly in that we place no restrictions on the motion of the UAVs, other than kinematics. Instead of computing the control input online in a receding horizon manner, we find the optimal (with respect to the fused geolocation error covariance) joint control policy for a long horizon using dynamic programming [2]. The utility of this approach is that we get to see, for the first time, the behavior of the optimally coordinated UAV trajectories.

In fact, the coordination observed in the optimal UAV trajectories is surprising in that the distance to the target dominates the advantage of diverse (i.e. $90^{\circ}$ ) viewing angles. The optimal coordination has the UAVs taking turns passing over the target. These results may guide the design of more practical heuristic controllers that are near-optimal for tracking.

The paper is organized as follows. In Section II, we discuss the video-based measurement model, the geolocation error covariance, and the UAV system dynamics. In Section III, the proposed dynamic programming approach is developed, including a quantized relative state space and cost function. Simulation results are presented in Section IV for two scenarios in which different target speeds are considered. Conclusions and directions for future work are discussed in Section V. 


\section{PROBLEM FORMULATION}

Consider a group of two UAVs tasked with estimating the state of a moving target vehicle. The UAVs fly at a fixed forward speed while maintaining a constant altitude. The target vehicle moves on the ground, and has a forward speed that can be significantly slower than that of the UAVs. Each UAV makes measurements of the target using a gimbaled video camera. The main objective is to optimize the coordination of the UAVs with respect to the joint estimation error covariance. It is assumed that UAVs communicate with a base station, where the fusion takes place. In this section, we first discuss the measurement model and the resulting geolocation error covariance. We then describe how the UAVs are modeled as discretized Dubins vehicles.

\section{A. Measurement Model}

Each UAV has a video sensor that makes image-plane measurements of the target. To relate these image-plane measurements to topographical coordinates, we closely follow the work of Mallick [1]. Video tracking uses two main coordinate frames: the topographic coordinate frame ( $T$ frame) and the sensor coordinate frame ( $S$ frame). The $T$ frame is the primary coordinate frame, and its $X, Y$, and $Z$ axes are along the East, North, and upward directions, respectively. The $S$ frame is the secondary frame and has its origin at the optical center of the camera. In the $S$ frame, the $Z$ axis is along the optical axis and is pointed in the general downward direction toward the Earth.

In following Mallick's methods, we also adopt his notation. Therefore we denote transposes with " ' ", estimated quantities with "^”, and random vectors with " ”. Also, superscript " $S$ " denotes a quantity in the sensor coordinate frame; however, where Mallick uses superscript " $T$ " on vector quantities to indicate that they lie in the topographic coordinate frame, we omit such notation.

To perform the coordinate transformation from the $T$ frame to the $S$ frame, we use the orthogonal attitude matrix $T_{T}^{S}(\boldsymbol{\theta})$, which is a nonlinear function of the Euler angles $\left(\boldsymbol{\theta} \in \mathbb{R}^{3}\right)$. We note that the Euler angles are those of the camera sensor and not the aircraft and that its inverse is denoted by $T_{S}^{T}(\boldsymbol{\theta})$. Moreover, we assume that image tracking software is available to control the camera's gimbal platform and keep the target in the camera's field of view. This software must also report the camera's Euler angles.

Let $\mathbf{s}:=\left[s_{x}, s_{y}, s_{z}\right]^{\prime}$ and $\mathbf{0}:=\left[o_{x}, o_{y}, o_{z}\right]^{\prime}$ represent the true sensor and ground target positions, respectively, measured from the $T$-frame origin. The relationship between the object and image point is defined through the attitude matrix,

$$
\mathbf{r}^{S}:=\mathbf{o}^{S}-\mathbf{s}^{S}=T_{T}^{S}(\boldsymbol{\theta})(\mathbf{o}-\mathbf{s}),
$$

and we let $r:=\left\|\mathbf{r}^{S}\right\|$ and $\mathbf{u}^{S}:=\mathbf{r}^{S} / r$. Here, $r$ is the range from the $S$ frame origin to the target position, and $\mathbf{u}^{S}$ is the unit vector along the camera's line-of-sight.

The relation between the sensor and target positions can be estimated as

$$
\hat{\mathbf{o}}=\hat{\mathbf{s}}+\hat{r} T_{S}^{T}(\hat{\boldsymbol{\theta}}) \hat{\mathbf{u}}^{S}
$$

The quantity $\hat{r}$ is computed using the flat-Earth approximation. To determine $\hat{\mathbf{u}}^{S}$ we need a model that relates the video sensor measurements to the world. Here, we use the perspective transformation, which accounts for the nonlinearities in this mapping. Let $\mathbf{p}:=\left[\begin{array}{ll}p_{u} & p_{v}\end{array}\right]^{\prime}$ denote the noisy pixel location corresponding to the $3 \mathrm{D}$ object point o. The noisy video measurement is modeled as

$$
\mathbf{p}=\mathbf{h}(\mathbf{o}, \boldsymbol{\eta})+\mathbf{n},
$$

where $\mathbf{h}$ is the nonlinear measurement function, $\boldsymbol{\eta}$ is the video camera parameters vector (it includes the extrinsic and intrinsic camera parameters), and $\mathbf{n}$ is a zero-mean Gaussian measurement noise with covariance $R$, i.e. $\mathbf{n} \sim N(\mathbf{0}, R)$. In practice, $\hat{\mathbf{u}}^{S}$ can be determined from the observed pixel coordinate and the camera parameters.

\section{B. Geolocation Error Covariance}

The quality of a particular measurement depends on the location of the UAV with respect to the target. When the UAV is far from the target, relative to its height, the estimation error covariance is intuitively elongated. Here, we again follow [1] in quantifying this covariance for the video measurements.

The main, uncorrelated sources of error arise from the estimates for sensor position $(\hat{\mathbf{s}})$, sensor attitude angles $(\hat{\boldsymbol{\theta}})$, and terrain height $\left(\hat{h}_{0}\right)$. Hence, the error models are:

$$
\begin{aligned}
\hat{\mathbf{s}} & =\mathbf{s}+\tilde{\mathbf{s}}, \tilde{\mathbf{s}} \sim N\left(\mathbf{0}, R_{\tilde{\mathbf{s}}}\right), \\
\hat{\boldsymbol{\theta}} & =\boldsymbol{\theta}+\tilde{\boldsymbol{\theta}}, \tilde{\boldsymbol{\theta}} \sim N\left(\mathbf{0}, R_{\tilde{\boldsymbol{\theta}}}\right), \\
\hat{h} & =h_{0}+\tilde{h}_{0}, \tilde{h}_{0} \sim N\left(0, \sigma_{\text {ter }}^{2}\right) .
\end{aligned}
$$

Given the estimated geolocation in (2), the corresponding geolocation error can be written as

$$
\tilde{\mathbf{o}}:=\hat{\mathbf{o}}-\mathbf{o}=\tilde{\mathbf{s}}+\hat{\mathbf{u}} \tilde{r}+\hat{r} \tilde{\mathbf{u}},
$$

where $\hat{\mathbf{u}}=T_{S}^{T}(\hat{\boldsymbol{\theta}}) \hat{\mathbf{u}}^{S}$. Linearizing the attitude matrix $T_{S}^{T}(\boldsymbol{\theta})$ about the operating point, we obtain a relation for $\tilde{\mathbf{u}}$ as follows:

$$
\tilde{\mathbf{u}}=T_{S}^{T}(\boldsymbol{\theta}) \tilde{\mathbf{u}}^{S}+C\left(\mathbf{u}^{S}, \boldsymbol{\theta}\right) \tilde{\boldsymbol{\theta}}+C\left(\tilde{\mathbf{u}}^{S}, \boldsymbol{\theta}\right) \tilde{\boldsymbol{\theta}},
$$

where

$$
C\left(\mathbf{u}^{S}, \boldsymbol{\theta}\right)=\frac{\partial\left(T_{S}^{T}(\boldsymbol{\theta}) \hat{\mathbf{u}}^{S}\right)}{\partial \boldsymbol{\theta}}
$$

is the linearization of the attitude matrix. Ignoring the higher order term $C\left(\tilde{\mathbf{u}}^{S}, \boldsymbol{\theta}\right) \tilde{\boldsymbol{\theta}}$ and the effects of the pixel location error manifested in $\tilde{\mathbf{u}}^{S}$, we can use the approximation:

$$
\tilde{\mathbf{u}} \approx C\left(\mathbf{u}^{S}, \boldsymbol{\theta}\right) \tilde{\boldsymbol{\theta}} .
$$

We now need to define a relation for the term $\tilde{r}$ in (7). Starting from the definition for the estimated object position (2), and using the flat-Earth approximation,

$$
\hat{r}=\frac{\hat{h}_{0}-\hat{s}_{z}}{\hat{u}_{z}} .
$$


By substituting $h_{0}+\tilde{h}_{0}$ for $\hat{h}_{0}, s_{z}+\tilde{s}_{z}$ for $\hat{s}_{z}$, and $u_{z}+\tilde{u}_{z}$ for $\hat{u}_{z}$, we obtain

$$
\tilde{r}=\frac{\tilde{h}_{0}-\tilde{s}_{z}}{u_{z}+\tilde{u}_{z}}-\left(\frac{h_{0}-s_{z}}{u_{z}}\right)\left(\frac{\tilde{u}_{z}}{u_{z}+\tilde{u}_{z}}\right) .
$$

Treating $\tilde{r}$ as a function of $\boldsymbol{\zeta}=\left[\begin{array}{lll}\tilde{h}_{0} & \tilde{s}_{z} & \tilde{u}_{z}\end{array}\right]^{\prime}$, and linearizing about $\zeta=0$ reveals

$$
\tilde{r}=\frac{\tilde{h}_{0}-\tilde{s}_{z}}{u_{z}}-\frac{h_{0}-s_{z}}{u_{z}^{2}} \tilde{u}_{z} .
$$

Now, by substituting (10) and (13) into (7), we obtain

$$
\tilde{\mathbf{o}}=B \tilde{\mathbf{s}}+\frac{\mathbf{u}}{u_{z}} \tilde{h}_{0}+r B C\left(\mathbf{u}^{S}, \boldsymbol{\theta}\right) \tilde{\boldsymbol{\theta}},
$$

where $B$ is a matrix defined by:

$$
B=I_{3 \times 3}-\mathbf{u}\left[\begin{array}{lll}
0 & 0 & 1 / u_{z}
\end{array}\right] .
$$

By computing the covariance of (14), we arrive at the geolocation error covariance,

$$
\begin{aligned}
P_{\tilde{\mathbf{o}}}=B R_{\tilde{\mathbf{s}}} B^{\prime}+ & \frac{1}{u_{z}^{2}} \mathbf{u u}^{\prime} \sigma_{t e r}^{2} \\
& +r^{2} B C\left(\mathbf{u}^{S}, \boldsymbol{\theta}\right) R_{\tilde{\boldsymbol{\theta}}} C^{\prime}\left(\mathbf{u}^{S}, \boldsymbol{\theta}\right) B^{\prime},
\end{aligned}
$$

where $R_{\tilde{\mathbf{s}}}, \sigma_{t e r}^{2}$, and $R_{\tilde{\boldsymbol{\theta}}}$ are the covariances for the sensor position, the terrain height, and the Euler angles, respectively.

The dominant source of error arises from the uncertainty in the sensor attitude angles. Moreover, we let $R_{\tilde{s}}=0_{3 \times 3}$ and $\sigma_{\text {ter }}^{2}=0$, in which case the geolocation error covariance from (16) simplifies to

$$
P_{\tilde{\mathbf{o}}}=r^{2} B C\left(\mathbf{u}^{S}, \boldsymbol{\theta}\right) R_{\tilde{\boldsymbol{\theta}}} C^{\prime}\left(\mathbf{u}^{S}, \boldsymbol{\theta}\right) B^{\prime} .
$$

Because tracking will be done in the ground plane, only the $2 \times 2$ upper-left submatrix of $P_{\tilde{o}}(k)$ is relevant,

$$
P_{i}=\left[\begin{array}{cc}
P_{\tilde{\mathbf{o}}: 1,1} & P_{\tilde{\mathbf{o}}: 1,2} \\
P_{\tilde{\mathbf{o}}: 2,1} & P_{\tilde{\mathbf{o}}: 2,2}
\end{array}\right] .
$$

With two UAVs collecting independent measurements of the target, the fused geolocation error covariance can be computed as

$$
P=\left(P_{1}^{-1}+P_{2}^{-1}\right)^{-1}
$$

\section{UAV Modeling}

The Dubins vehicle is a planar vehicle that moves forward at a fixed velocity and has a bounded turning radius. It is commonly used to provide a simple model for UAVs flying at a fixed altitude. We assume that all UAVs are flying at a constant speed of $v_{a}$, and that they have a bounded turning rate, $\omega_{\max }>0$. For an individual agent $i$, let $\left(x_{i}, y_{i}, h\right)$ denote its absolute position in the $T$ frame, where $h$ is fixed, and let $\psi_{i}$ denote its heading. Then the kinematics of the UAV are described by

$$
\begin{aligned}
\dot{x}_{i}(t) & =v_{a} \cos \left(\psi_{i}(t)\right) \\
\dot{y}_{i}(t) & =v_{a} \sin \left(\psi_{i}(t)\right) \\
\dot{\psi}_{i}(t) & =u_{i}(t),\left|u_{i}\right| \leq \omega_{\max } .
\end{aligned}
$$

We assume that the $i^{\text {th }}$ UAV and its camera sensor have the same position, $\mathbf{s}_{i}=\left[x_{i}, y_{i}, h\right]^{\prime}$.
Solutions to the path planning problem are usually done in continuous time. However in coordinated target tracking, discrete-time dynamics are appropriate because:

1) Communication constraints, sensor sampling rates, and onboard computing power limit the frequency of target geolocation updates,

2) Discrete motion primitives are an efficient solution to computing optimal trajectories for Dubins vehicles [3].

To discretize the Dubins vehicle dynamics, we apply a zeroorder hold $(\mathrm{ZOH})$ on the control input, with a sampling time of $T_{s}$. For nonzero input, the equations of motion become

$$
\begin{aligned}
x_{i}^{+} & =\frac{v_{a}}{u_{i}}\left[\sin \left(u_{i} T_{s}+\psi_{i}\right)-\sin \left(\psi_{i}\right)\right]+x_{i} \\
y_{i}^{+} & =\frac{v_{a}}{u_{i}}\left[\cos \left(\psi_{i}\right)-\cos \left(u_{i} T_{s}+\psi_{i}\right)\right]+y_{i} \\
\psi_{i}^{+} & =u_{i} T_{s}+\psi_{i},
\end{aligned}
$$

whereas for zero input, we have

$$
\begin{aligned}
x_{i}^{+} & =v_{a} T_{s} \cos \left(\psi_{i}\right)+x_{i} \\
y_{i}^{+} & =v_{a} T_{s} \sin \left(\psi_{i}\right)+y_{i} \\
\psi_{i}^{+} & =\psi_{i} .
\end{aligned}
$$

\section{DYNAMIC PROGRAMMING}

In general, dynamic programming can address a wide class of applications comprised of many different physical systems described by dynamical equations of motion that require optimized trajectories. Here, we apply the technique of dynamic programming to produce an optimal joint control policy for a pair of UAVs tracking a target. We first present the state space and quantization required for tractability, and then describe the cost function.

\section{A. State Space and Quantization}

The computational cost of solving a dynamic programing problem is highly sensitive to the dimension of the state space. While each UAV requires three states and the target requires additional states, the dimension of the state space is reduced by considering the positions of the UAVs relative to the target,

$$
\mathbf{z}_{i}(k)=\left[\begin{array}{c}
x_{r}(k)-x_{i}(k) \\
y_{r}(k)-y_{i}(k) \\
\psi_{i}(k)
\end{array}\right], i=1,2 .
$$

Here $\left(x_{r}, y_{r}, 0\right)$ is the absolute position of the target (or reference) in the $T$-frame and $k$ is the discrete time index. The individual relative coordinates can be stacked to form the state $\mathbf{z}(k)=\left[\mathbf{z}_{1}^{\prime}(k), \mathbf{z}_{2}^{\prime}(k)\right]^{\prime}$.

Correspondingly, the planar distance of the $i^{\text {th }}$ agent to the target,

$$
d_{i}(k):=\sqrt{z_{i, 1}^{2}(k)+z_{i, 2}^{2}(k)},
$$

will play a vital role in the UAV control policy. Here, $z_{i, 1}(k)$ and $z_{i, 2}(k)$ are the first two entries of $\mathbf{z}_{i}(k)$. Moreover, $d_{i}(k)=0$ implies that agent $i$ is directly above the target at an altitude of $h$. 
The UAV platform under consideration travels at a fixed speed of $v_{a}=15 \mathrm{~m} / \mathrm{s}$, and the UAVs communicate to a fusion center at a frequency of $1 \mathrm{~Hz}$. Consequently, the sampling time $\left(T_{s}\right)$ is one second. To make the optimization feasible, we quantize the control and state spaces. Optimal Dubins paths use only three inputs, so we let

$$
\begin{aligned}
\mathcal{U} & :=\left\{-\omega_{\max }, 0, \omega_{\max }\right\} \\
u_{i}(k) & \in \mathcal{U}, i=1,2,
\end{aligned}
$$

with $\omega_{\max }=0.5$. In quantizing the state space, note that with $T_{s}=1$ and $\omega_{\max }=0.5$, the UAV headings will be well approximated by

$$
\begin{aligned}
\Psi & :=\{0.5 n\}_{n=0}^{12} \\
\psi_{i}(k) & \in \Psi, i=1,2 .
\end{aligned}
$$

To quantize the relative positions, let $z_{\max }>0$ denote the boundary of the relative spatial coordinates and $q>0$ denote the quantization step between each of the coordinates,

$$
\begin{aligned}
\mathcal{Z} & :=\left\{-z_{\max }+q n\right\}_{n=0}^{2 z_{\max } / q} \\
z_{i, 1}(k) & \in \mathcal{Z}, \forall i=1,2 \\
z_{i, 2}(k) & \in \mathcal{Z}, \forall i=1,2 .
\end{aligned}
$$

The parameters $z_{\max }$ and $q$ should be selected to balance the fidelity of spatial resolution with practical considerations such as time and computer memory.

\section{B. Cost Function}

The cost function we use for dynamic programming is designed to produce UAV trajectories that optimize the estimation performance over a long planning horizon. Here, the estimation performance is quantified by the trace of the fused geolocation error covariance (19). The resulting cost function is evaluated over a planning horizon of $N_{s}$ stages (time instances) according to

$$
V_{0}(\mathbf{z})=\frac{1}{N_{s}} \sum_{k=0}^{N_{s}-1} L(\mathbf{z}(k)),
$$

where

$$
L(\mathbf{z}(k))=\operatorname{trace}(P(\mathbf{z}(k))),
$$

is the instantaneous cost in which the fused covariance is evaluated with respect to the relative coordinates.

The cost function in (25) is based entirely on the objective of gathering the "best" joint measurements. The joint measurement error covariance (19) is incorporated into the cost rather than the target state estimate error covariance, as the use of the latter would yield a problem incompatible with dynamic programming. Nonetheless, optimizing measurements can be interpreted as optimizing state estimates for the case in which process noise dominates measurement noise.

Dynamic programming uses backwards induction to find the optimal joint control policy, $\Pi_{k}(\mathbf{z})$, which maps each relative state $\mathbf{z}$ at time $k$ to the optimal steering input for each UAV. We apply a standard technique known as value iteration in which the optimal cost to go is computed using the recursion

$$
V_{k}(\mathbf{z})=\min _{\mathbf{u} \in \mathcal{U}^{2}}\left(L(\mathbf{z}(k))+V_{k+1}(f(\mathbf{z}, \mathbf{u}))\right), \forall \mathbf{z},
$$

from $k=N_{s}-1$ to 0 with $V_{N_{s}}(\mathbf{z})=0, \forall \mathbf{z}$. At time $k$, the input obtaining the minimum in (27) for each $\mathbf{z}$ is stored in $\Pi_{k}(\mathbf{z})$. Here, $f(\mathbf{z}, \mathbf{u})$ is the relative dynamics derived from (21) by assuming a particular (known) target motion.

\section{SIMULATION RESULTS}

We now demonstrate the effectiveness of dynamic programming applied to the optimal coordinated target tracking problem. Two cases are considered. In the first, the target has a slow speed compared to that of the UAVs, whereas in the second, the target is moving faster. In both cases, the target has a constant velocity along the $X$-axis at zero altitude. The second scenario is used to compare the performance of the optimal controller to Kingston's splay state controller [6]. The simulation parameters are summarized in Table I.

We simulate continuous UAV trajectories using the $N_{s^{-}}$ horizon optimal controller $\Pi_{0}(\mathbf{z})$. The input for the nearest quantization point is applied and held over a single sampling period, after which the control is recomputed. Note that with dynamic programming, the optimal control policy is independent from the initial conditions.

\section{A. First Scenario}

In the first scenario, the target travels at a speed of $5 \mathrm{~m} / \mathrm{s}$, and we optimize over a horizon of $N_{s}=90$ seconds. UAV trajectories resulting from an arbitrarily chosen initial state are shown in Figure 1. Corresponding planar distances to the target, $d_{1}$ and $d_{2}$, the relative angle $\gamma$, and the instantaneous cost, $L(\mathbf{z})$, are shown in Figure 2.

In Figure 1, we observe the UAVs making alternating passes over the target. It is apparent from Figure 2 that the two UAVs do not maintain a $90^{\circ}$ relative angle to the target. Although the UAVs do occasionally pass though a $90^{\circ}$ relative angle, the trace of the fused error covariance has its minimum values when at least one UAV is on top of the target, i.e., $d_{1}(t)=0$ or $d_{2}(t)=0$. For example at the times of 20 and 40 seconds, the values of the trace are comparatively lower than at other time instants. Thus, we conclude that the optimal controller maneuvers the UAVs so that one is always close to the target, independent of the relative viewing angles. Similar behavior is observed from any initial condition.

\begin{tabular}{|c|c|c|c|}
\hline Parameter & Description & Value & Units \\
\hline $\mathrm{h}$ & UAV Altitude (AGL) & 100 & $\mathrm{~m}$ \\
\hline$v_{a}$ & UAV Speed & 15 & $\mathrm{~m} / \mathrm{s}$ \\
\hline$v_{r}$ & Target Speed & $\{5,7\}$ & $\mathrm{m} / \mathrm{s}$ \\
\hline$N_{s}$ & Planning Horizon & $\{90,60\}$ & $\mathrm{s}$ \\
\hline$T_{s}$ & Sampling Time & 1 & $\mathrm{~s}$ \\
\hline$R_{\tilde{\boldsymbol{\theta}}}$ & Sensor Attitude Covariance & $9 I_{3 \times 3}$ & $\mathrm{deg}^{2}$ \\
\hline$\omega_{\max }$ & Max. Turning Rate & 0.5 & $\mathrm{rad} / \mathrm{s}$ \\
\hline$z_{\max }$ & Max. UAV-Target Distance & 75 & $\mathrm{~m}$ \\
\hline$q$ & Quantization Step & 5 & $\mathrm{~m}$ \\
\hline
\end{tabular}

TABLE I: Simulation Parameters 


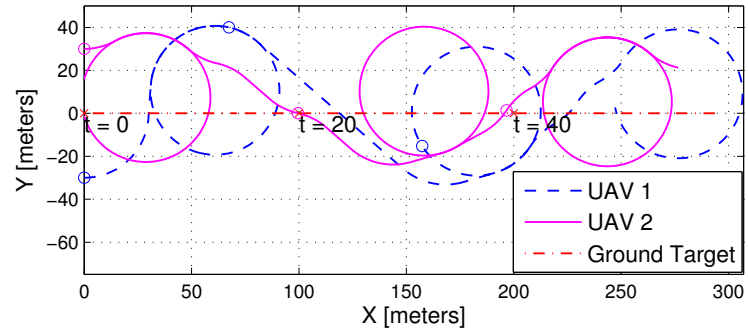

Fig. 1: Trajectories for two UAVs tracking a single target with a speed of $5 \mathrm{~m} / \mathrm{s}$.
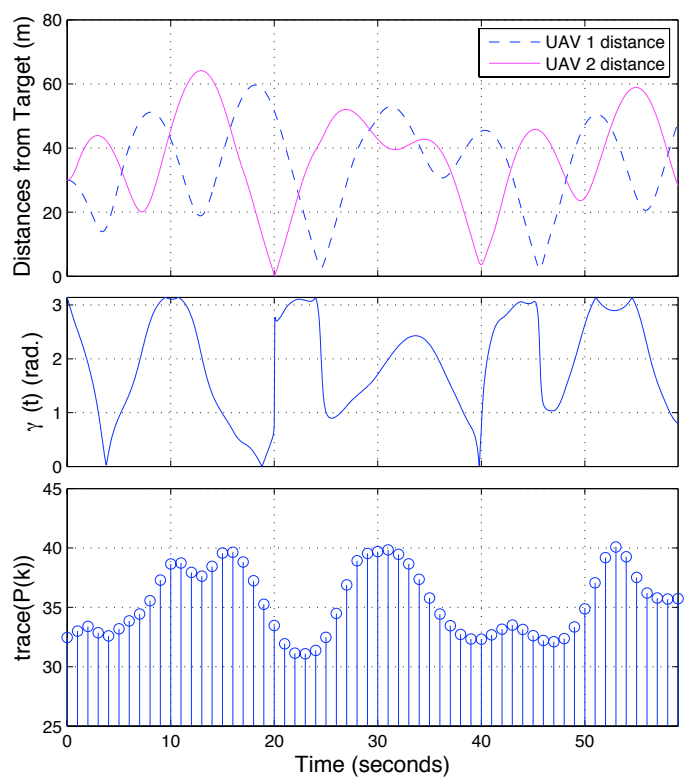

Fig. 2: Distances, relative angle, and instantaneous cost for the optimal trajectories shown in Fig. 1.

\section{B. Second Scenario}

In the second scenario, the target travels at a speed of 7 $\mathrm{m} / \mathrm{s}$, and we optimize over a horizon of $N_{s}=60$ seconds. The purpose of this second scenario is twofold. First, we want to see if the UAV behavior is dominated by angle or distance coordination for a target moving faster than in the slow target scenario. Second, we want to compare the optimal UAV trajectories to those coming from a well-established coordinated controller. Here, we choose to compare to the splay state controller from Kingston [6], with the nominal orbit radius set to $65 \mathrm{~m}$. This orbit radius is the distance the UAVs should maintain from the target.

The typical behavior resulting from the application of these two controllers is depicted in the UAV trajectories shown in Figure 3. Corresponding data is shown for the optimal controller in Figure 4 and for the splay state controller in Figure 5. For Kingston's controller, the distance to the target is not shown in Figure 5 as it stays within $3 \mathrm{~m}$ of the set point.

The first observation regarding the optimal UAV trajectories is that they now have a different shape. Instead of

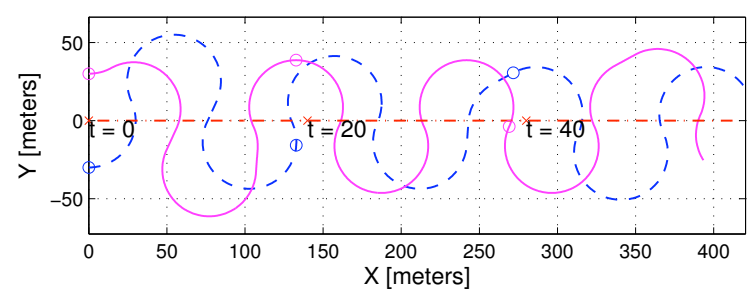

(a) Dynamic Programming

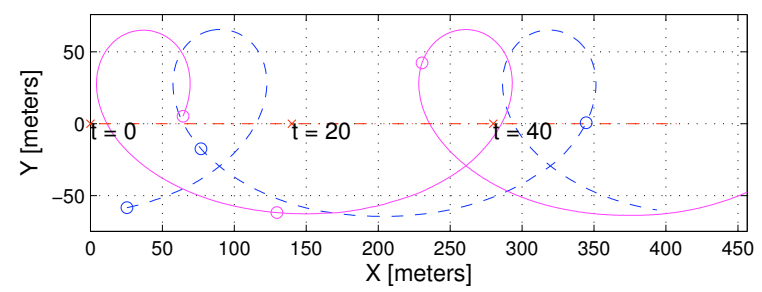

(b) Splay State Formation Controller

Fig. 3: Comparison of trajectories generated by (a) dynamic programming and (b) the splay state controller. In this scenario, the target travels at $7 \mathrm{~m} / \mathrm{s}$.

the combination of circling and straight motions observed in Figure 1, the UAVs now make back-and-forth motions over the target. In analyzing Figure 4, it is again apparent that distance is the dominating factor in the optimal coordination.

This point is further apparent in comparing the instantaneous cost between the optimal controller and the splay state controller. The splay state controller maintains an angle separation near $90^{\circ}$ throughout the simulation. However, the trace of the geolocation error covariance is significantly higher than that of the optimal controller because the UAVs stay far from the target. Moreover, even though a $90^{\circ}$ separation angle is advantageous in reducing the trace of the error covariance, its benefit is overshadowed by that of having at least one UAV as close to the target as possible. Thus we again conclude that distance coordination dominates angle coordination.

\section{Static Interpretation}

To gain insight into the observed distance-dominated coordination behavior, we setup two stationary scenarios in which the first UAV is fixed at distance of $d_{1}=50 \mathrm{~m}$ from the target. We then plot the trace of the fused geolocation error covariance as a function of $d_{2}$ and the relative angle, $\gamma$, for two different UAV altitudes (see Figure 6). When the UAVs are low, relative to the distance to the target, the error covariance ellipses are significantly elongated. In this case, orthogonality between viewing angles is advantageous, but primarily when $d_{2}$ is large. When the UAVs fly at a more reasonable altitude, the effect of orthogonal angles is unnoticeable. Of course, these are static scenarios, and dynamic programming effectively brings the motion constraints into the UAV routing optimization.

\section{CONCLUSIONS AND FUTURE WORK}

Dynamic programming proves to be effective in optimizing the coordination of two camera-equipped UAVs for the 


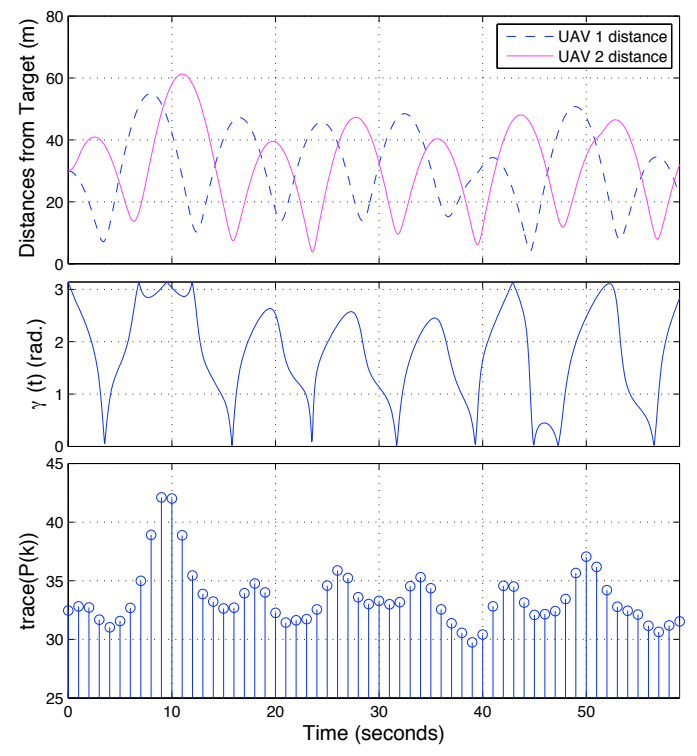

Fig. 4: Distances, relative angle, and instantaneous cost for the optimal trajectories shown in Fig. 3(a).

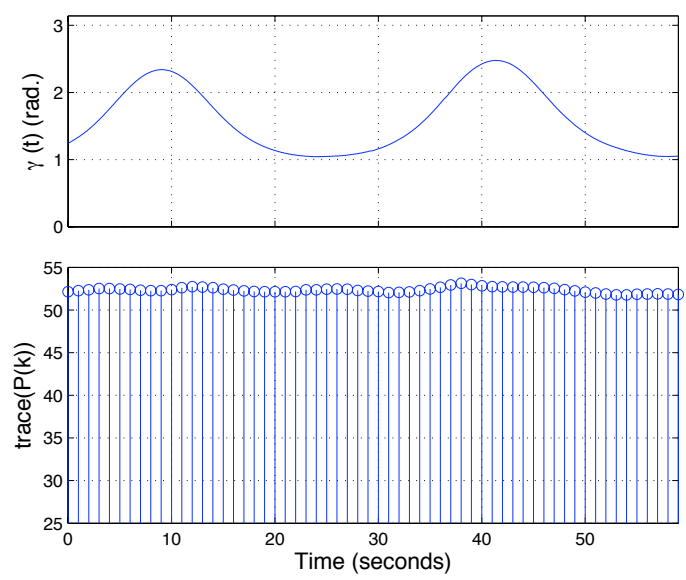

Fig. 5: Relative angle and instantaneous cost for the splaystate trajectories shown in Fig. 3(b). The UAVs maintained a distance of $65 \pm 3 \mathrm{~m}$ from the target.

purpose of tracking a target. With the optimal controllers in place for given target kinematics, the behaviors of the UAVs are shown to be governed by their planar distances to the target rather than their separation angle. This result was interpreted through a static analysis in which the separation angle and distance to target were varied parametrically. When one UAV is close to the target, its estimation error ellipse is nearly circular, so the relative angle to the other UAV is irrelevant.

Future work involves addressing more complicated target motion and accommodating larger UAV fleets. Regarding variable target motion, changes in target direction can be handled via coordinate transformations while changes in target speed can be addressed by switching between several control policies, each of which is optimized for small ranges

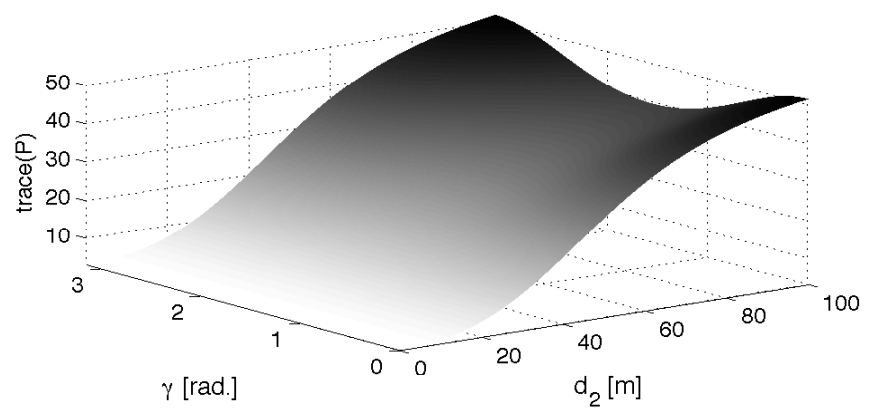

(a) UAVs at 25-m altitude

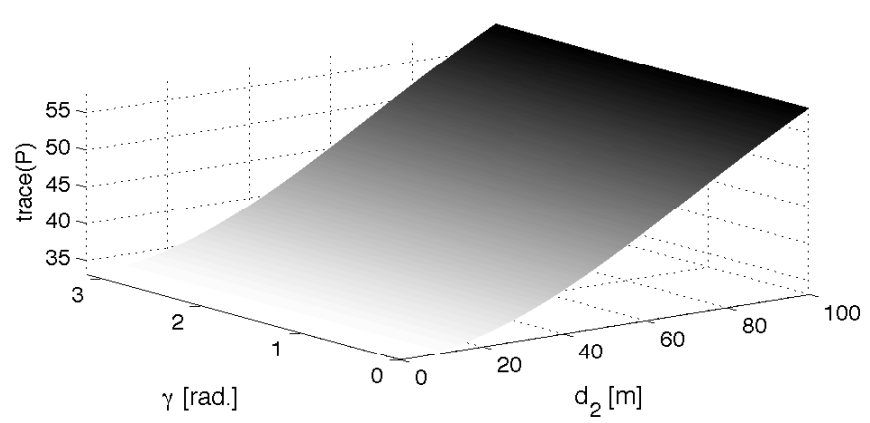

(b) UAVs at $100-\mathrm{m}$ altitude

Fig. 6: Effects of distance from target versus angles between UAVs.

of target speeds. To employ larger UAV fleets, we plan to develop heuristic feedback controllers that achieve nearoptimal performance guided by the distance-coordination behavior observed in the optimal controllers.

\section{REFERENCES}

[1] M. Mallick. Geolocation using Video Sensor Measurements. Proceedings of the $10^{\text {th }}$ International Conference on Information Fusion. Quebec, Canada: IEEE, 2007.

[2] F. Lewis and V. Syrmos. Optimal Control. 2nd ed. NY: John Wiley \& Sons, Inc., 1995.

[3] X.C. Ding, A. Rahmani, and M. Egerstedt. Multiple-UAV Convoy Protection: An Optimal Approach to Path Planning and Coordination. IEEE Transactions on Robotics, (to appear).

[4] E. Lalish, K. A. Morgansen, and T. Tsukamaki. Oscillatory control for constant-speed unicycle-type vehicles, in Proc. 46th IEEE Conf. on Decision and Control, New Orleans, Louisiana, USA, 2007.

[5] E. W. Frew. Sensitivity of Cooperative Geolocalization to Orbit Coordination, in AIAA Guidance, Navigation, and Control Conference, Hilton Head, SC, Aug. 2007.

[6] D. B. Kingston. Decentralized control of multiple UAVs for perimeter and target surveillance, Ph.D. dissertation, Brigham Young University, Provo, Utah, December 2007.

[7] D. J. Klein and K. A. Morgansen. Controlled collective motion for trajectory tracking, in American Control Conference, Minneapolis, Minnesota, USA, June 2006.

[8] R. A. Wise and R. T. Rysdyk. UAV Coordination for Autonomous Target Tracking, in Proceedings of the AIAA Guidance, Navigation, and Control Conference, 2006.

[9] M. Mallick and B. F. La Scala. Differential Geometry Measures of Nonlinearity for the Video Filtering Problem. Proceedings of SPIE. Orlando, Florida, 2008.

[10] M. Stachura, A. Carfang, and E. W. Frew. Cooperative Target Tracking with a Communication Limited Active Sensor Network. International Workshop on Robotic Wireless Sensor Networks. Marina Del Rey, CA, June 2009.

[11] G. Gu, P. R. Chandler, C. J. Schumacher, A. Sparks, and M. Pachter Optimum cooperative UAV sensing based on Cramer-Rao bound, in Proceedings of the American Control Conference, 2005. 